\title{
Morgagni hernia: Ten years of idiopathic vomiting
}

\author{
Ahmed A. Mustafa Khalil* and Aim M. McMillan
}

\section{INTRODUCTION}

Morgagni hernia was first described in 1769 by the Italian anatomist Morgagni (1). This hernia is a rare congenital diaphragmatic hernia comprising $2-3 \%$ of all diaphragmatic hernias $(1,2)$. The bowel, stomach, omentum or even the liver can herniate into the chest through a congenital retrosternal defect where the diaphragm joins the costal arch, as first described by Larrey, Napolean's chief surgeon (3).

A very small defect is gradually stretched with time, explaining why many cases are silent until adulthood, and presentation may vary from non-specific gastrointestinal symptoms to bowel obstruction and strangulation $(2,4)$. Gastric outlet obstruction is often the presenting picture when the stomach herniates into the sac $(5,6)$.In childhood, pneumonia is an important presentation $(7,8)$. More than half of patients can be diagnosed incidentally while investigating unrelated problems and most symptomatic cases tend to present acutely (9). Despite careful investigations for various presenting symptoms, diagnosis is unsuspected until incidental finding of the hernia on laparotomy or laparoscopy (9).

We report a patient who was thoroughly investigated over ten years for repeated vomiting. Repeated chest radiographs, endoscopies, oesophageal manometry and barium follow through revealed no abnormalities. Finally, a CT scan obtained to rule out chronic pancreatitis, showed the hernia and excluded pancreatitis. Subsequently, laparoscopic mesh repair allowed for rapid recovery.

\section{METHOD}

A 35 year old male presented with unsettling vomiting

*To whom correspondence should be addressed:

Ahmed A Mustafa Khalil

Department of surgery

Queen Margaret Hospital

Dunfermline

KY12 0SU

Email: aamk72@doctors.org.uk for seven days. He suffered attacks of vomiting and dysphagia for ten years with rapid deterioration and 12.5 kilograms weight loss in the past six months. Over the years, he was treated with different proton pump inhibitors and Metoclopromide orally with little response. The patient recognises that liquid food can improve his symptoms, and bread can aggravate his vomiting. He had a recent history of pale stool for three month that was difficult to flush as well as heavy alcohol consumption for several years.

His past medical history includes depression, asthma, latex allergy, right inguinal hernia repair when he was 12 years old and persistent vomiting for 10 years following main meals, no haematemesis, and weight loss in the past six months.

His full blood count, urea and electrolyte, liver function test and calcium levels were within normal range. Chest radiograph reported right basal consolidation and atelectasis with loss of demarcation of the diaphragm (Figure 1).

Upper GI endoscopy revealed oesophagitis, hiatus hernia, a small oesophageal nodule at $38 \mathrm{~cm}$, and a normal stomach and duodenum. Histopathology revealed an eosinophilic nodule consistent with reflux oesophagitis.

A manometric study excluded dysmotility and reported reduced tone in the lower oesophagus.

The patient had two normal upper GI endoscopies in the past on one occasion, however, an hour glass looking stomach was reported, but no obstruction was noted.

A small bowel follow through was requested to investigate steatorrhoea, revealing an unusual lie of the stomach position with a degree of malrotaion. Otherwise the examination was unremarkable (Figure 2).

A CT scan of the abdomen showed a normal pancreas, and a $5 \times 8 \mathrm{~cm}$ defect in the right hemi-diaphragm representing a Morgagni hernia. The sac contains half of the stomach body, transverse colon and mesentery, with no evidence of bowel obstruction (Figures 3 and 4). 


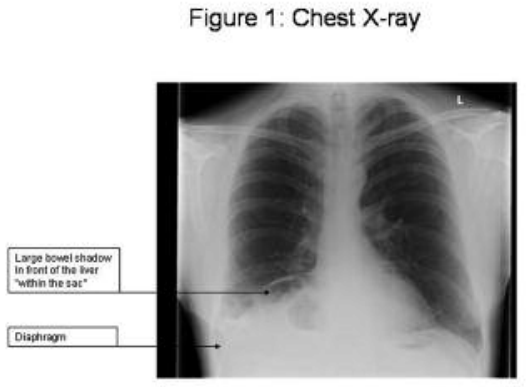

Figure 1: Chest X-ray

Figure 3: CT scan (transverse section, Chest)

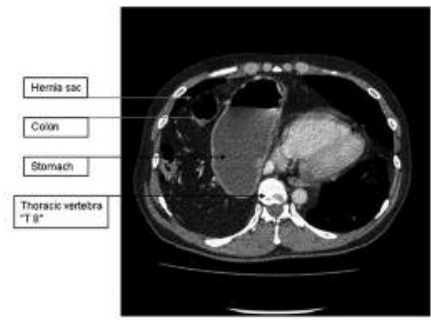

Figure 3: CT scan (transverse section, Chest)

On laparoscopy, the colon and stomach were pulled down with ease from a wide sac that extended to the right side of the chest. Laparoscopic excision of the sac was performed by dissecting the plane between the peritoneal sac and the pleura which easily pealed off. The diaphragmatic defect was repaired with a $10 \times 15 \mathrm{~cm}$ composite mesh (Bard ${ }^{\circledR}$ Composix Mesh $\AA$ ) and was fixed in place with Auto Suture (ProTackTM).

\section{DISCUSSION}

Although the bulk of reports confirm that Morgagni hernia often remains silent and the majority of cases are discovered incidentally while investigating unrelated problems, the majority of symptomatic cases present acutely with potentially life threatening obstruction and strangulation (9). Pallati et al presented a case of gastric outlet obstruction, diagnosed as Morgagni hernia involving the stomach at laparotomy (5).

Lateral view chest radiographs can be helpful and may show an anterior mediastinal mass typically to the right of the midline. Iso et al presented a case of acute intestinal obstruction with air fluid level in the chest and right side of the chest on plain X-rays of the abdomen and chest (10). In children, it might only show features of consolidation. However, the diagnosis can be missed

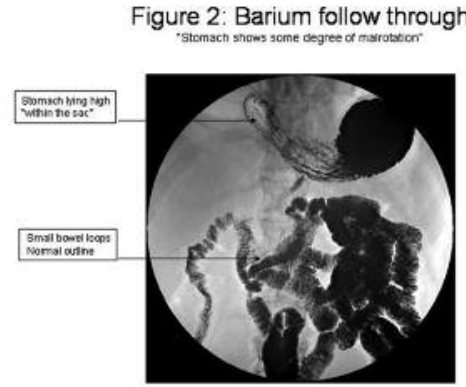

Figure 2: Barium follow through

"The stomach shows some degree of malrotation"

Figure 4: CT scan (coronal section)

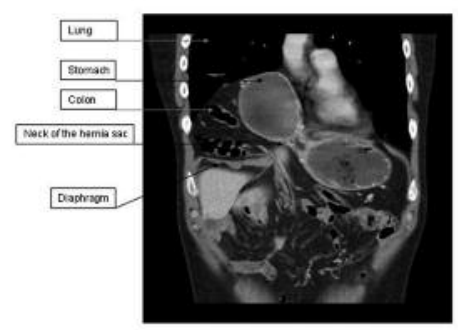

Figure 4: CT scan (coronal section)

(7) and in the patient in this case presentation, the chest radiograph did not raise a suspicion of a diagnosis of Morgagni hernia.

Contrast studies can be completely normal (9) and usually depend on whether any bowel or stomach herniates at the time of the study. This was the case in the patient presented in this case report, and a barium follow through was not helpful in the diagnosis.

Nearly all cases of gastric outlet obstruction will necessitate an upper GI endoscopy. However, in the patient in this case report, endoscopy was not conclusive on three occasions. This may reflect either intermittent herniation or reduction of the stomach while inflating or inserting the endoscope. Dodis et al reported that endoscopy was also inconclusive in his case report (11).

Although this patient was symptomatic for ten years a CT scan and operative findings confirmed the presence of a large hernia with colon and stomach content, all other investigations had been unsuccessful. This case demonstrates the difficulty of diagnosing patients with a Morgagni hernia. While many cases have been diagnosed on chest radiographs and CT scans, the patient presented in this report remained undiagnosed until a CT scan was requested to rule out another 
diagnosis. The CT scan demonstrated both the extent and contents of the hernia and identified its anatomical location. Thus a CT scan is the diagnostic method of choice and can confirm up to $100 \%$ of cases, it can differentiate the hernia from other mediastinal masses and chest pathologies as well as provide a detailed description of the diaphragmatic defect and hernia sac, including its contents (12-14).

The defect is usually small and situated to one side of the midline $(2,15)$. It is likely that this patient had an intermittent herniation through the unusually wide neck with strangulation and initiated repeated episodes of gastric outlet obstruction. Furthermore, it is possible that the stomach had repeatedly been reduced back into the abdomen before diagnostic imaging was performed. The most recent presentation, which prompted the diagnosis, may have reflected an attack of incomplete gastric volvulus that led to intense vomiting and dysphagia.

\section{CONCLUSION}

Morgagni hernia is a rare diaphragmatic hernia. It is usually asymptomatic, but can present with a nonspecific symptoms, posing a challenge to its diagnosis. Patients with long-standing symptoms of gastric outlet obstruction and negative routine radiographs and endoscopy, should be suspected for a diagnosis of Morgagni hernia. Early CT scan should be considered as it is both sensitive and specific.

\section{REFERENCE}

1. Morgagni GB. The seats and causes of diseases investigated by anatomy. London: Miller and Cadell 1769;3:205.

2. Harrington SW. Clinical manifistation and surgical treatment of congenital types of diaphragmatic hernia. Rev Gastroenterol;18:243.

3. John H, Juhl M, editors. Paul and Juhl's Essentials of Radiologic Imaging: Lippincott Williams \& Wilkins (LWW); 1998.

4. Sakalkale RP, Sankhe M, Nagral S, Patel CV. Obstructed Morgagni's hernia (a case report). J Postgrad Med 1991;37(4):228B, 229-30.

5. Pallati PK, Puri V, Mittal SK. Gastric outlet obstruction secondary to Morgagni hernia: a case report. Hernia 2008;12(2):209-12.

6. Turut H, Demirpolat G, Bulbuloglu E, Yuksel M. Lifethreatening vomiting caused by large Morgagni hernia in an octogenarian. Asian Cardiovasc Thorac Ann 2008;16(3):240-1.

7. Al-Salem AH, Nawaz A, Matta H, Jacobsz A. Herniation through the foramen of Morgagni: early diagnosis and treatment. Pediatr Surg Int 2002;18(2-3):93-7.

8. Lev-Chelouche D, Ravid A, Michowitz M, Klausner JM, Kluger Y. Morgagni hernia: unique presentations in elderly patients. J Clin Gastroenterol 1999;28(1):81-2.

9. Loong TP, Kocher HM. Clinical presentation and operative repair of hernia of Morgagni. Postgrad Med J 2005;81(951):414.

10. Iso Y, Sawada T, Rokkaku K, Furihata T, Shimoda M, Kita J, et al. A case of symptomatic Morgagni's hernia and a review of Morgagni's hernia in Japan (263 reported cases). Hernia 2006;10(6):521-4

11. Dodis LB. Gastroduodenal Obstruction From Morgagni Hernia in an Elderly Patient.7 (4):70.

12. Anthes TB, Thoongsuwan N, Karmy-Jones R. Morgagni hernia: CT findings. Curr Probl Diagn Radiol 2003;32(3):135-6.

13. Yildirim B, Ozaras R, Tahan V, Artis T. Diaphragmatic Morgagni hernia in adulthood: correct preoperative diagnosis is possible with newer imaging techniques. Acta Chir Belg 2000;100(1):31-3.

14. Minneci PC, Deans KJ, Kim P, Mathisen DJ. Foramen of Morgagni hernia: changes in diagnosis and treatment. Ann Thorac Surg 2004;77(6):1956-9.

15. Craighead CC, Strug LH. Diaphragmatic deficiency in the retrocostoxiphoid area. Surgery 1958;44(6):1062-9.

\footnotetext{
Ahmed A Mustafa Khalil is a specialist registrar in General surgery at the Perth Royal Infirmary in Scotland, U.K. Aim Macmillan is an upper GI consultant and general surgeon at the Queen Margaret Hospital in Dunfermline, U.K.
} 